\title{
The concept of a model of plastic bodily image in architecture
}

\author{
Sergey Malakhov ${ }^{1, *}$ \\ ${ }^{1}$ Samara State Technical University, Institute of Architecture and Civil Engineering, 194, \\ Molodogvardeiskaya St., 443001, Samara, Russia
}

\begin{abstract}
One of the key problems of architectural mastery is lack of acute feeling of the plastic image and bodily self-determination of an architectural object at the initial (and the subsequent) stage when the design model author is trying to see and understand the context where the future object should appear, literally be born out of thin air. This sensuous amnesia is caused, among other reasons, by the lack of experience in "sculptural modeling", "hand molding" and today's common practice of facilitated transfer to analytical computer design. The loss of intense bodily experience, mental connection of one's own body with an imaginary object, has an effect as well. In its turn, it deprives the object of sensuous corporeal nature, transforms it into a mechanistic conglomerate. The article deals with the body concept, bodily and plastic categories in relation to the architectural shaping and suggests the concept of mediator models linking reality and the designer's imagination integrated into a new typology of "models of plastic bodily images" (MPBI). The principal medium of these models and the procedures for their creation are based on synthesis of form, interpretation of the "bodily experience", tactile contact with model material, sculptural forming techniques and sensory evaluations of subjectenvironment interaction. The proposed typology of models has been piloted by the author in numerous educational and conceptual projects. The results of experiments and developed theoretical principles related to models of plastic bodily images will help achieve better results in the course of basic design and special composition training of architects.
\end{abstract}

\section{Introduction}

A number of contemporary authors persistently draw their attention to tactile experience that is disappearing not only from architectural practice, but also from the life-style of a modern city dweller.

Richard Sennett in Flesh and Stone reflects on the problem of "sensory deprivation" the loss of "bodily experience" in the architecture of modern cities. He sees the reason in the increased degree of simulation of the physical reality associated with our bodily experience, or the experience "of our body." The reason for imitative behavior that ignores physical sensuality lies in a particular role of media proposing to replace real experience

\footnotetext{
*Corresponding author: s_a_malahov@mail.ru
} 
with imaginary ... "This desire to free the body from resistance is coupled with the fear of touching [1] ..."

Juhani Pallasmaa [2], Alexander Ermolaev [3], Steven Holl [4] confirm the importance of manual modeling, developing designer's tactile skill, experience in sculpting and drawing, live contact with "sculptural body of architectural objects". The role of artistic intuition, the link between architectural form and bodily experience, one's own body form are actively communicated in creative concepts of Le Corbusier [5], Thom Mayne [6] Frank Gehry [7]. The concepts of physicality are considered by nonclassical postmodern philosophers (Michel Foucault. [8], R. Barthes. [9], M. Merleau-Ponty [10], and others.).

The concept of sculptural image is a universal issue in art related to works of a sculptor, as well as choreographer, and architecture here is interpreted across different types of art. For example, A. Kantor [11] defines plasticity as "artistic expression of a spatial form", but it also has a broader interpretation. Attempts to deeper understanding of physicality in relation to architecture are evident in the study of Richard Sennett [1], certain aspects of which are related to the content of this article.

General principles of organic architecture are addressed in studies and theoretical concepts of Frank Lloyd Wright [12] and further on studies in works of Alvar Aalto [13], Rafael Moneo [14], Sou Fujimoto [15].

Generalized preliminary sketches of architectural objects are quite common in architectural practice. However, there is a significant lacuna in understanding the importance and special features of an image, as if it is informing the outside observer that an autonomous entity with sensual bodily nature, just as soulful as the natural landscape itself, is entering the current environment. There should appear subtle, but quite distinct manifestation in architectural form - echoes, remnants of bodily sculptural reflection. And not all "generalized sketches" can fulfill the role. In theory of architectural methodology such preliminary or subsequent images (or "proto-objects") are not given sufficient attention to, the very concept of this type of models is absent as such.

The purpose of this article is to update the issue of preliminary (or post-project) modeling of the plastic bodily image (the plastic body), to describe the concepts of corporeal and plastic in the context of architecture, and to justify the typology and methodology of establishing the corresponding models.

The following questions should be answered: 1) does a plastic body exist in architecture, or is it just an assumption, a kind of mental mirage? 2) how does plastic bodily reflection manifest itself in the images of the body (or "proto-object")?

\section{Materials and Methods}

If you take simple rectangular shapes we are accustomed to use based on the functional block diagrams of designing software and imagine how they are integrated into the landscape, one experiences a vague impression that the rectangles suffer from a deficit of contact with the smooth surface of horizontal terrain. Rectangle is too aggressive cutting the smooth surface, and the bigger it is, the more aggressive form it appears to take. For example, a small rectangular house completely blends in with the natural environment, but a more expanding object ceases to resonate with all sorts of curves: both in sections in plane view. Rural farmsteads of Russian province traveled along the slope surfaces heading towards the river and utilized gently curved planning vectors. Therefore it is not so difficult to interpret this principle within a graphic sketch by aligning one box to the next, just like a train, but using smooth turns. At the moment when the farmstead reaches its critical value, it ceases to exist as a strict rectangular shape and acquires an image of a living body responsive to the terrain. 


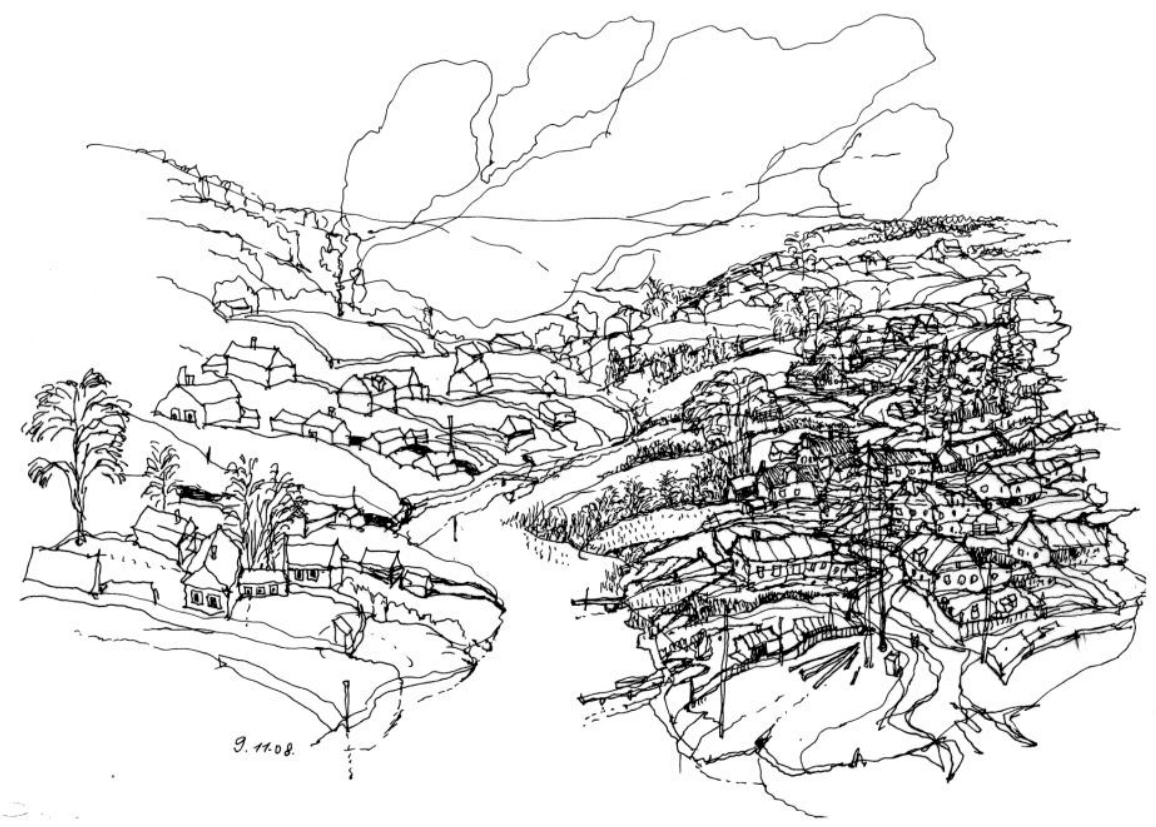

Fig. 1. Rhythmic and plastic harmony of Russian provincial landscape

Sketches interpreting the theme of "liveliness" of the strict geometric shapes and their architectural adaptation to natural landscape are improvisational in nature and carry out the function of a mediator between the reality of a built farmstead and the organic form. The sketch itself, be it in graphic form, or an architectural model, or a product of inner vision, can be called "a plastic body" of an architectural object, i.e. a materialized image of an organic form that is more abstract, or resembling a project sketch.

The existence of such mediator models ("proto-objects") helps the designer produce a generic and specific representation of the object with the principles of plastic urban landscape harmony being accentuated: a system linking buildings and nature in a single architectural image.

Mediator models, "models of plastic bodily image" establish the connection between the objective and the environment. But these sketches are not the ones combining the functional elements of the program. First, these models are designed to articulate sensual characteristics of a would-be object, its "plastic body" setting stage for going further into detail. In experiments dedicated to projects of locations and settlements, models illustrating the idea of the locality as an architectural plastic body were being created over the course of several years (2010-2016). These models preceded detailed designs both at the level of the general plan (mega-object) and during the development of individual (forming) objects [16]. What are the concepts in other discourses that can be related to models in question?

First, these models correlate with the concepts of "plastic" and "bodily", formulated in philosophy and art history.

A body in postmodern philosophy is perceived as a phenomenological consequence of the connection of a human body and their soul, that is, the human body with all its physical, sensory, and somatic states serve as origins in the discourse on physicality.

According to T. K. Kerimov, "physicality is understood not as an object, not as a set of organs, but as a specific unity, the unthought horizon of human experience which constantly exists prior to any particular reasoning. Not fit for reflective analysis, indecomposable following the scheme of consistent rational actions, physicality turns to be original in relation to natural and cultural objects, which exist because of it, and of which 
that they are the expressions"... [17]." "As a result, the very idea of one's "inner world" has been reconsidered, as with introduction of the concept of "body consciousness" the distinction between "inner" and "outer" appeared to be non-existent, at least in theory. This is a fairly common scientific myth of modern philosophical reflection which spawned a wide range of various theoretical speculations. Suffice it to recall Merleau-Ponty's "phenomenological body" as a specific kind of "being of the third type" ensuring a constant dialog of human consciousness with the world and thus - the sensory-semantic integrity of subjectivity, says Karimov.

Merleau-Ponty argued that "the focus of meaning" and mimetic values the world is endowed with is the human body. Merleau-Ponty proposes that the source of any meaning lies within animate human body inspiring worlds and forming together with them a "correlative unity." [17].

Does this thesis confirm the fact that phenomenal body can serve as the embodiment of architectural designs in the form of models establishing direct, associative or indirect relationship with the human body? Expanding the influence of human body on the process of generation of any other idea, Merleau-Ponty characterizes physicality, rising from the spiritualized human body, as "a phenomenal body," "system of possible actions", "potential body" phenomenal location of which is determined by the objective and the situation [10]. " Architectural models expressing the unity of material and spiritual substantiality, sensual and tactile nature of the form, may also be included in the discourse of Merleau-Ponty's phenomenal body as they predetermine "a system of possible actions" with the object, and these actions are preconditioned by "the phenomenon of position" (in its broad meaning, of course).

In contrast to the non-classical philosophy (post-modernism), which dissociates itself from direct analogies with the physicality of human body, architectural models of plastic bodily images, the concept of which is put forward in our study, include a wide range of discourses on physicality: 1) visual presence and redefining the body image; 2) mediated transfer of bodily experiences to form (erotic component, for example); 3) immanent experience of hands; 4) transfer of bodily experience and perceptions of a body into the concept of space, houses and city; 5) a harmonious urban landscape form.

Postmodern research of physicality regarded it as a socio-public (M. Foucault [8]) and cultural-semiotic phenomenon (R. Bart [9]).

All these five discourses of plastic bodily images are synthesized in the act of creating a model. These models can be created before or after the architectural projects. Chaotic ("careless") Frank Gehry's sketches generally address the sculptural image including author's personal associative links [7]. Modern interpretations of the idea of architectural body are presented in the works of Thom Mayne [6] and Maciek Jozefowicz [18]. In these models, the body itself and physicality are related as sensually controlled generalized clusters of "architectural mass" and the processes of sensual control over form.

"Indeed, the art of the XX century, especially its second half, and above all the artifacts and art practices of Post Culture, demonstrate the build-up of some overwhelming bodily world perception (see: Suprematism)

"... contemplation gives way to a haptic, tactile in the broadest sense, relation of the subject with the art object. Touching and feeling on all levels (from primitive tactile, through visual to mental) now rules in the field of artistic and aesthetic experience [17]. "

Body and physicality can be viewed as conditions predetermining the creation of architectural form of an object at the preliminary stage, when the details or related objects are not yet specified [35]. Not without interest is the estimation of sculptural and physical characteristics of an object at the stage of post-project analysis, including cases studying the existing areas (fragments) of urban landscape. 
Bodily principle of these models is embodied by means of articulation of the object boundaries, mass concentration, the choice of the dominant type of physicality: monumental or organic; integration of these types; identifying associative image and character of the opposition (integration) of the architectural body and the environment. The associative parallel with the human body serves as the foundation for bodily effect, which, at different stages of architecture development, manifested itself in varying degrees of convergence of the architectural form and its prototype: from symbolic in antiquity - to direct in pots modernism.

Lying, reclining human figure, as well as closely pulled together group of human bodies, are directly attached to harmonious natural landscape, and are therefore repeated in a flattened (concentrated) model of plastic bodily image of urban environment [16].

Richard Sennett addresses the issue of complex relationship of body and architecture in his study [1]. Investigating the design and semantic history of Roman architecture of Hadrian times, Sennett sees a similarity between these objects and pantomime. Sense of life is leaving the architecture; object body prevails over the lost essence of a city for the sake of imperial self-image and certain cosmogonic installations. The human body is losing its value and is replaced with the scenery and staging. A cosmogonic body is born and further on communicated to military camp design and the Pantheon. Roman city starts from the point called the umbilicus Urbis, corresponding to human body navel, from this point, two main streets of a Roman city originate: Decumanus Maximus and Cardo Maximus. At this point, the altar for the higher forces was laid called Mundus, it also served as a starting point for setting the external border of the city - Pomerania.

According to the pagan tradition, the highest body communicated to the city arises from the analogy with the human body. Navel for a man, considered Vitruvius [20], plays as important of a role as umbilicus for the Roman city. "... not without a reason did geometry prompt similar biological analogies", says Sennett, "Despite the huge size of the Pantheon, it is impossible to escape the feeling that the building acts as an extension of the human body $[1,123] . "$ However, Sennett's body is not just anatomical, it is even more than just a "higher body". For him, body is a kind of filter through which state the author examines the prospects for living in the city and, furthermore, the city as a sign of civilization capable or not capable of cooperative and rightful way of life.

Formally speaking, the plan and spatial composition of Rome in Hadrian era depicts maximum alignment of architecture with the idea of physicality. What could be more corporeal than the massive stones separated from each other by the layout of street canyons, hidden behind the external borders of the forum, the Colosseum arena, atrium space, oikos and tricliniums? Moreover, all these shapes, perceived as impenetrable masses (bodies), were constructed on an accurate geometric and symbolic basis. The curves of planning axes in the famous Villa Adriana [20] only reinforced the image of a particular geometrical and physical microcosm as they allowed each of the following objects (architectural bodies) to further articulate their self-sufficiency by breaking the seemingly continuous "urban grid", its division into formal clusters enclosing the next geometrically adjusted pattern.

In simple terms, these were contrasting circles, squares and rectangles; in the sophisticated images we distinguish arenas, peristyles, basilicas (The New Map of Rome by Giovanni Battista Nollie, 1748 (Roman Antiquities by Giovanni Battista Piranesi, 1756; [21]). The way of a spectator ("the observer of architectural bodies") runs through a system of hierarchically subordinate routes, fractal arrangement of an ordered saying, the repeated "message" magnified the effect of the planned performance (theatrum mundi). On the other hand, planning axes rotation in the Roman forums is likely to have been forced by the need to squeeze the "Roman grid" into the space between the Quirinal, the Capitol and the Palatine. Etienne-Louis Boullee [21] most physically and aphoristically illustrates the key objects of the revolutionary Paris in the form of monumental masses with extremely 
generalized texture of stone walls. On top of that, these massive bodies serve as shells concealing the "absolute emptiness".

The analysis of plastic bodily component within the overall spatial (urban) city matter is a key apology of "absolute architecture" in Mathias Ungers' and Aldo Rossi's works [21], [22], and Pierre Vittorio Aurelli study [21]. Corporality thus implies categorical mass (emptiness) which opposes the indifferent urban matter (Adrian Boullee, Ungers); or the organic integration of the architectural body (Wright, Thom Mayne).

Of course, Sennett, concerned about "sharing painful experience" ("... to live in exile together" [1, 465]), is primarily interested in restructuring the ethical paradigm of urban community. But even in such a minor, by his standards, action as "bodily form" arrangement, there is a significant positive factor: the study of new physical forms of locations embodying the binary basis of effective architectural and functional organization.

\section{Results}

The new form, inheriting the idea of corporeality with all its synthesized components, should incorporate the initially divided and contradictory intentions: for example, the limited (inviolable physical shell, Pomerania) and the outbreaks; the speed and tactile contact; individuality and collaboration; the house and the city.

The concept of mediator models, or "models of plastic bodily image" performing the function of "protoobjects" and inheriting the two paradigms of "bodily plastic form" presented above, is proposed for thorough architectural understanding of this issue: the "absolute" and the "organic architecture."

Here, a proto-object is something followed by the object itself in the chain of design procedures. Without the stage of defining the plastic body, it generalized image, truly heartfelt (anticipated) "spacial need", the following "decoding of elements" can come in confrontation with the surrounding landscape, both artificial and natural.

Since the role of a plastic body can and should be performed by an imaginary or discovered form, derived from the experience of physically created models (following the principle of internalization of activity: you can not conceive before you do!), we have to name some of the principles that characterize models of plastic bodily images.

1. The principle of substantiality of plastic bodily models: models of plastic bodily images are classified as "models of substantial form," i.e., they reproduce their own qualities of form without purposeful attitude towards functional context.

2. The principle of generalization of form and outward perception of models of plastic bodily images: the medium of models of plastic bodily images is a generalized presentation of possible forms of the architectural object perceived by external viewer and focused on the harmonization of visual relationship of the object and the environment.
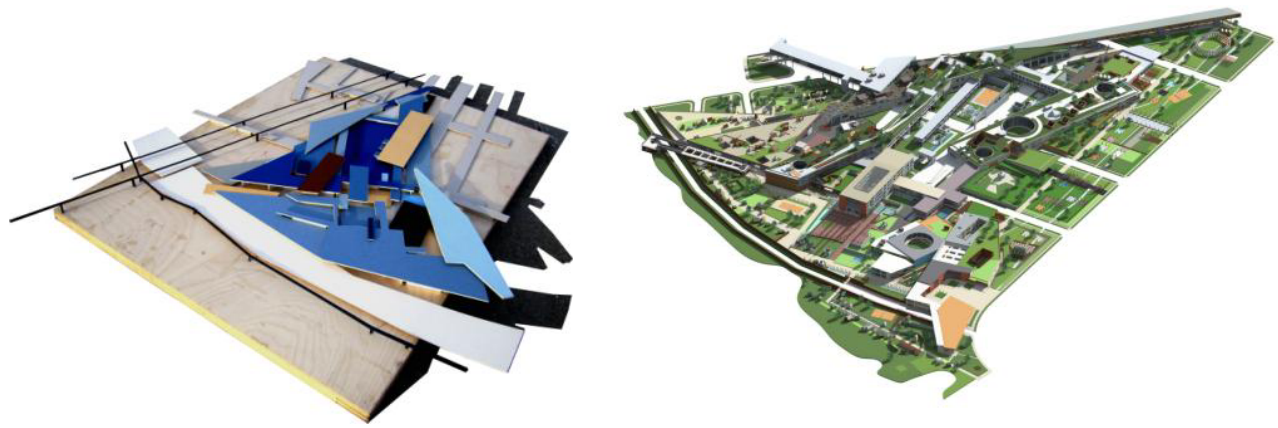

Fig. 2. Plastic bodily model preceding mega-object project design 
3. The principle of physicality of plastic bodily models: the physicality of models manifests itself in reporting the accomplished act of sculptural feeling, covering models with the author's hands, locking its shape, "weighting" the model above the base surface, giving it relative autonomy of all autonomous bodies identifying their own unexpressed object mass.
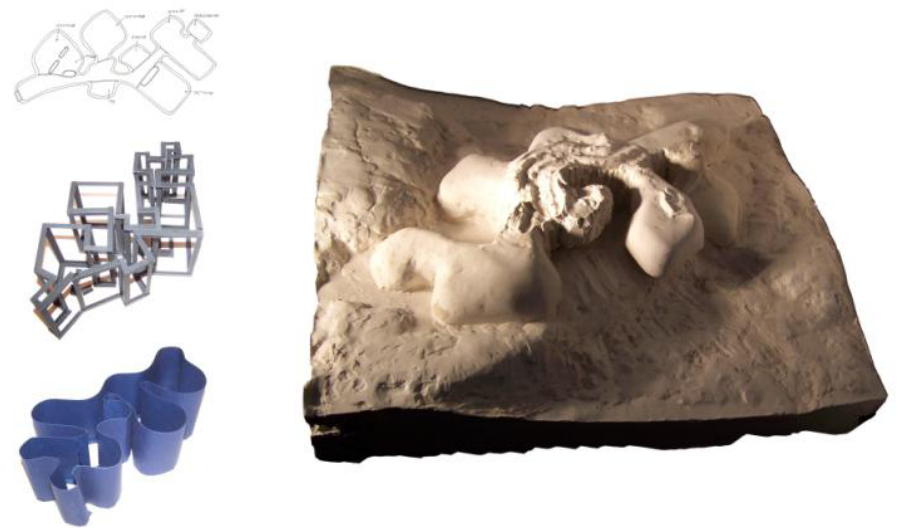

Fig. 3. Design of plastic bodily models with plastic materials as the first stage of object spatial modeling

4. The principle of necessary plasticity of the models of plastic bodily images: the models plasticity is achieved through the complex deviation of simple geometric shapes dating back to clay crafting or utilizing other material that obeys formative or erotic impulses of a hand prompted, in turn, by their own intuition transfered from the depths of sensory-physical body experience. Increase in size of the plastic form object leads to deviation from straight lines so that no incompatibility with the landscape and curved horizon appeared later on. A person needs plasticity to take architecture back to natural forms associated with those of nature.

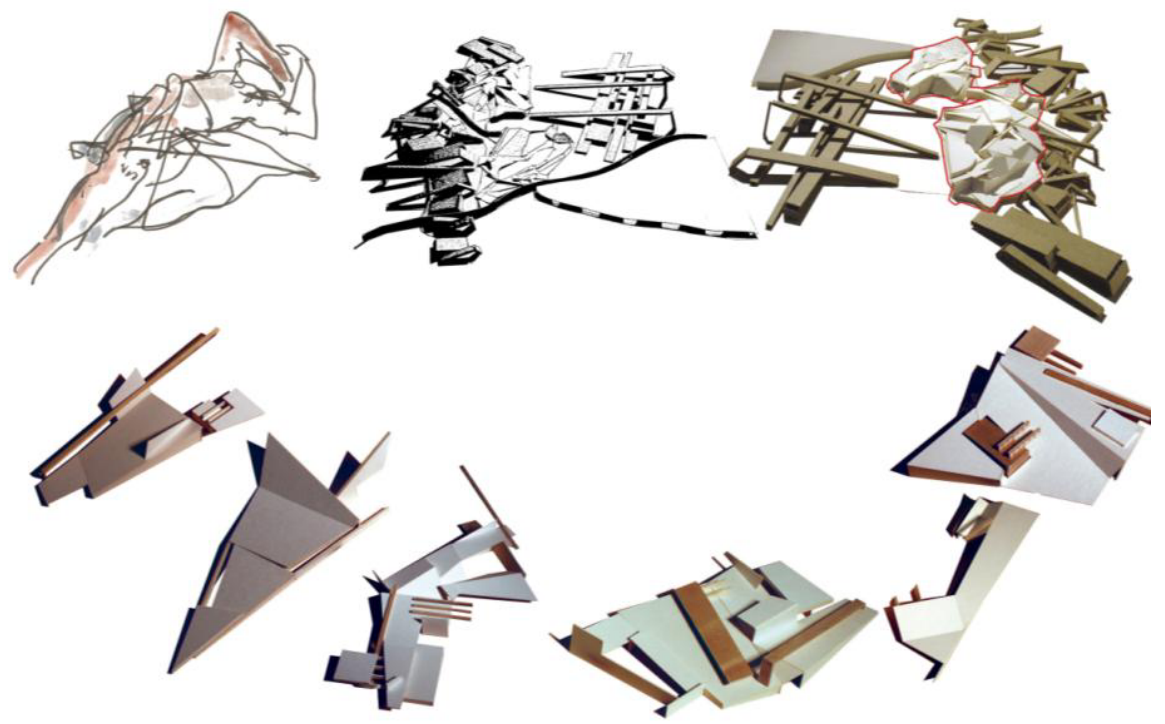

Fig. 4. Creating Models of Plastic Bodily Image at The Stage of Preliminary Design of Urban Landscape Fragment (Mega-objects and Forming Objects). 


\section{Discussion}

Farrelly addresses the importance of general plastic modeling stage: "An abstract drawing of a city can be a more efficient way to give an impression of the urban quality. In this case, the urban site can not be seen but the drawing helps the viewer to understand that there are dynamic forces at play within the space." [23]. (In: Farrelly, L. (2011) p.27). B. Wipper perceives physicality, concentration on generalized masses as common ground of architecture and sculpture [24]. Nicholas Olsberg discusses the connection between sculpture and architecture [25]. Delicate, "emotional" "feeling," "modeling" in pencil or watercolors of the nature object explored by the artist gives us one more reason for understanding the meaning of models of bodily image. John Burdger's sketch with irises demonstrates tactile sensitivity of form and, at the same time, its integrity as a sculptural body perfectly matching the concept of models of plastic bodily images [26]. To a certain degree, Hartman and Keil study highlights plastic modeling technique in graphics [27]. In the book Design and Analysis, published by Delft professors, different variations of threedimensional analytical charts resembling models of plastic bodily images are presented [28]. The question of body and bodily experience in architecture is complicated by theoretical discussion of the sculptural notion. Immediately, there is a dilemma: how far from each other are the concepts of sculptural and plastic?

Explanations offered by philosophical dictionaries suggest that the term sculptural refers to visual arts, whereas plastic may describe a particular quality of form associated with smooth lines reminding us of the beauty and particulars of a human body and natural landscape. Additional meaning of plasticity of form comes from soft, flexible, smooth materials modeled by sculptor's hands. These materials primarily include clay, play dough, wax, oil paint brush strokes. Accordingly, the model implements the idea of plastic form if the form is related to the properties mentioned above.

\section{Conclusions}

The above mentioned four basic principles of models of plastic bodily images determine the general attitude towards a model of a generalized image of the architectural object, its configurational, plastic and tactile properties determined by bodily experience of connection of form and landscape. Other principles of formation related to fields of composition and language, and are considered by the author within general theory of composite design method [29], [30], [31], [32], [33], [36]. These other principles should include "the principle of binary opposition" "RI index", predetermining, among a series of actual oppositions, the relationships of "physical" and "structural" form; complex and simple, mass and voids and so on. Accordingly, searching for the image of opposition and dialog (compromise) between the "monumental" and "organic" corporeality of models of plastic bodily images follow the same principle (see e.g. works of Peter Eisenman [34].

The proposed typology of models of generalized form has been tested in numerous experiments in author's educational, conceptual and real-life projects.

The principles of creating models of plastic bodily images described in the article will improve the effectiveness of general training of architects in the field of composition and formation, and should link educational program and theories with the task of enhancing the harmony of urban landscape integrity.

\section{References}

1. R. Sennett, Flesh and Stone. The Body and the City in Western Civilization (2016) 
2. J. Pallasmaa, The Thinking Hand. Existential and Embodied Wisdom in Architecture (John Wiley and Sons Publication, England, 2009).

3. A. P. Ermolaev, Essays on Realities of Architect-Designer Profession. Names, judgments, analysis (Architecture-S, 2004)

4. S. Holl, Parallax (Birkhauser-Publishers for Architecture, Basel-Boston-Berlin, 2000)

5. Le Corbusier, Secrets of Creativity: Between Painting and Architecture. 1887 - 1965 (A.S. Pushkin Museum, Moscow, 2012)

6. Th. Mayne, A. Vidler, Morphosis. Buildings and Projects, 1993-1997 (Rizzoli, NY, 1999).

7. F. O. Gehry \& Associates, Inc. Frederick R. Weisman Art and Teaching Museum University of Minnesota. The Architecture Collection, V. 3, 134-143 (Victoria The Images Publishing, Australia, 1998)

8. M. Foucault, A History of Insanity in the Age of Reason (Book of Light, St. Petersburg, 1997)

9. R. Barthes, Selected works. Semiotics. Poetics (Univers, Progress, 1994)

10. M. Merleau-Ponty, Phenomenology of Perception (Juventas, Nauka, St. Petersburg, 1999)

11. A. M Kantor, The Subject and Medium in Painting (Tallinn, 1998)

12. A. F. Goldstein, Frank Lloyd Wright (Stroyizdat, Moscow, 1973)

13. R. Weston, Alvar Aalto (Phaidon, 1999).

14. V. Canals, S. Rush, M. Fraile, Rafael Moneo, The Architects of the City. Biennale Arhitettura 2012. Common Ground, 108-109 (Marsilio, Venice,, 2012)

15. S. Fujimoto, Architectural Works 1995-2015 (TOTO Publishing, Tokyo, 2015)

16. S. Malakhov, E. Repina, Interconnection of Project Tasks. Experiment on Innovative Design, Part 1 (NPO House of Art, Samara, 2011)

17. T. K. Kerimov, Modern Philosophical Dictionary (Academic Project, Moscow, 2015)

18. Saatchiart.com. Construction 1. "Lost in the Wildness". Reconstructed Sculpture by Maciek Jozefowicz

19. Vitruvius, Ten Books on Architecture (Architecture-S, 2006)

20. E. Bacon, Design of Cities (Penguin Books, 1976)

21. P. V. Aureli, The Possibility of an Absolute Architecture (2014)

22. A. Rossi, The Architecture of the City (2015)

23. L. Farrelly, The fundamentals of architecture (2011)

24. B. Vipper, Introduction to the Historical Study of Art (1985)

25. N. Olsberg, Architecture and Sculpture: A Dialog in Los Angeles, in The Architectural Review, march (2014)

26. J. Burdger, Bento's Sketchbook. How Does the Impulse to Draw Something Begins? (AdMarginem Press, 2012)

27. Unusual Architectural Presentation Drawings (Anja Hartman and Uta Keil, PAGE ONE, Berlin, 2004)

28. B. Leupen, Ch. Grafe, N. Kornig, M. Lampe, P. de Zeeuw, Design and Analysis (010 Pablishers, Rotterdam, 1997)

29. S. A. Malakhov, E. A. Repina, Training Workshop of Sergey Malakhov and Evgeniia Repina. 1999-2014 (TATLIN, Yekaterinburg, 2014) 
30. S. A. Malakhov, Proceedings of Samara Scientific Center of the Russian Academy of Sciences, V. 15, 2, 249-255 (2013)

31. S. A. Malakhov, K. E. Nikonov, The Concept of Day Gilbert Ecological Settlement (TATLIN, Yekaterinburg, 2014)

32. World Encyclopedia: Philosophy (AST, Harvest, Modern Writer, 2001)

33. S. A. Malakhov, K. E. Nikonov, GIEPOLIS. A harmonious and Efficient Settlement. Samara (Samara, 2014).

34. Eisenman Architects. Greter Columbus Convention Center. The Architecture Collection, V. 3, 52-61 (Victoria The Images Publishing, Australia, 1998)

35. S. A. Malakhov, Urban Construction and Architecture 4, 37-42 (2015). doi: 10.17673/Vestnik.2015.04.5.

36. E.O. Smolenskaya, Urban Construction and Architecture 1, 16-20 (2011). doi: 10.17673/Vestnik.2011.01.3. 